\title{
Cultural Pervasiveness or Objective Violence?: Three Questions about KOS as Cultural Arbiters
}

\author{
Richard P. Smiraglia \\ Knowledge Organization Research Group \\ School of Information Studies \\ University of Wisconsin, Milwaukee \\ smiragli@uwm.edu
}

\begin{abstract}
Knowledge organization and knowledge organization systems are pervasive in human experience, yet the effect of this pervasiveness is overlooked and little analyzed. Several authors have called for a theory of knowledge organization that embraces cultural and social realities alongside domain-centric ontologies. Examples of leading studies point to pervasive and occasionally oppressive discourses embracing race, sex and gender and economics. Three research questions are presented about how to study knowledge organization systems as cultural arbiters and how to incorporate temporality and atemporality into the methodology of subject ontogeny.
\end{abstract}

\section{Keywords}

Culture, Knowledge organization, Knowledge organization systems, culture, canons, warrant, discourse, subject ontogeny

\subsection{CULTURAL PERVASIVENESS, OBJECTIVE VIOLENCE: WHOSE CANON IS IT?}

Knowledge organization (KO) is pervasive in human experience-every act of cognition holds the possibility of categorization of perceived observations. Each act of cognition includes the act of sorting, every phenomenon is recognized by its perceived characteristics and therefore recognized as much for what it is not as for what it is. In real life, orderings are produced from grouping such cognitive conclusions. From spices in the kitchen to desk in-boxes to school pupils sorted by aptitude to local and express trains, ordering by categorization is absolutely pervasive. Jacob called this activity categorization, because it results in simple but useful groupings; such cognitive categorization activity can be

Advances in Classification Research, 2015, November 7, 2015, St. Louis, MO, USA. formally separated from the process of classification derived ontologically. Beghtol called the process naïve classification, particularly but not exclusively when it emerged from scholarly activity. Researchers sort data by their initial observations - older versus newer, longer versus shorter, treatment versus control-eventually these sorts of scholarly categorizations lead to new testable hypotheses. The testing of these hypotheses leads to even more data categorization and the emerging ordering is a form of naïve classification. Over time the classes, formerly simple categories but expanded by precise definition and inclusion-exclusion principles, become a naïve classification.

That such ordering activity is pervasive is commonly understood but not adequately analyzed in the $\mathrm{KO}$ domain. Pervasiveness means ubiquity and that means there is constant potential for social effect. It is at this point that classification's consequences (Bowker and Star 1999) become paramount. Social effect of classification is as critical for the $\mathrm{KO}$ domain to analyze and evolve theoretical positions about as is the continued perfection of concept theory or ordering techniques. Much research shone light on the potential of classifications to bring harm through their epistemic stances (e.g., Tennis and Adler 2013), especially those that incorporate points of cultural bias. Tennis (2013) presented an analytical framework for discovering points of "objective violence," which is to say, harmful positions in classifications that can lead to oppression whether by inference, direct action or neglect. The social classification of race known as apartheid, effectively described by Bowker and Star as among the more consequential forms of classification is well understood to have brought oppression.

But what about the child who browses shelves of school library books arranged according to the Dewey Decimal Classification and from the ordering discovers that homosexuality is classed as aberration, or that special classes exist to isolate works about women in roles formerly reserved to men? The point of view taken away by the child observer is both formative and informative. The child might be oppressed by the observation, or the child might have learned that such oppression is the norm. 
Either way, the pervasiveness of classification and its cultural affect is not well understood or documented in the $\mathrm{KO}$ domain.

Already in this essay the examples of knowledge organization systems (KOSs) extend well beyond the realm of the well-known bibliographic classifications. The domain of $\mathrm{KO}$ embraced a shift from knowledge as pure concept - ontology - to knowledge as experienceepistemology (Mai 2011), and then again to the perception of knowledge as lived experience-social epistemology (Smiraglia 2014). The straight-forward assignment of phenomena to mutually exclusive classes gave way to more nuanced understanding and therefore fuzzier boundaries as social effects were added to the calculus. Yet, the domain still waits to escape its obsession with general, so-called "universal" bibliographical classifications. All the while the functional world connected to a dream of a semanticallyhyperlinked system is focused on the evolution of domain specific ontologies. Are these KOSs? Of course they are. $\mathrm{KO}$ as a domain needs to turn its attention away from library shelving and look instead toward a future where ever more sophisticated hyper-linkages create ever new pathways to the use and evolution of knowledge.

Nevertheless a few key cases of cultural analysis stand as beacons for the domain. Furner, for example (2007, 1478), writing about race, described a distinction between natural kinds and nominal kinds. Natural kinds are entities that are classified by their elemental characteristics (Furner uses "water" as an example) whereas nominal kinds are classified by cumulative social perspectives. Viewed in this way social distinctions such as race or gender can be seen as ontologically variable along socially-perceptive trajectories. Thus KOSs that incorporate such nominal kinds are themselves ontologically variable.

Mai has suggested this approach incorporating traditional taxonomic observations with socially-constructed concepts such as nominal kinds to create a general theory of classification $(2011,717)$ :

A robust theory of classification is one that does not separate between how things really are and people's cognitive constructions of how things are; it is one that does not separate between the ontology of things and the epistemology of how we get to know about things. In other words, we need to develop a late-modern theory of classification-as-epistemology.

There are other approaches to the problem of the consequences of the cultural pervasiveness of classification. Semiotic theory tells us that everything has the potential to be perceived as a sign given meaning in the moments of perception. Pluralities of meaning are the result, and in classification theory we have arrived at a theory of facets to allow expression of pluralities of meaning around a specific entity or phenomenon.

We have begun to understand knowledge as more than the texts of documents and rather as lived experience. The KOS is an agent of social control of lived experience, and thus it is an arena for cultural dissemination. Dissemination means not just giving out, but also gatekeeping — not just spreading seed, but making sure the seed spread is culturally acceptable. Lived experience guarantees a certain volatility in that which is considered appropriate for cultural dissemination.

This paper is based on ideas first expressed in Cultural Synergy in Information Institutions (Smiraglia 2014) and it also is an extension of the foreword to Lee (2016). Lee's book shows in intimate detail the workings of a classification that was not derived primarily as an objective scheme for retrieval, but rather was intended completely to encourage a particular manner of lived experience in Chinese imperial society. The Seven Epitomes, originating about 7 or 6 BCE (Lee 2016, 23), was the first classified catalog and formal bibliography disseminated by Imperial China (Lee 2016, 1). Lee's text revealed the underlying purpose of the KOS enshrined in the Seven Epitomes (Lee 2016, 30):

For a period of two millennia, it acted as a decisive force in shaping scholarship and was valued as an authoritative version of the early Chinese intellectual history, although its roles in scholarship and intellectual history have also attracted criticism in the last century. One unique feature of the catalog worth special mention is its intellectual activism. Unlike its modern-day counterpart, the Seven Epitomes was never intended by its creator Liu Xin to be an objective information organization and retrieval tool. Instead, Liu Xin unabashedly pronounced his scholarly opinion and Classicist ideology through the words and classificatory structure, with an intent to sway and guide others. "

In that foreword, I wrote that where we cite literary warrant as the bulwark of cultural values enshrined in KOSs we see it as elemental in the discourse from culture to the KOS, but we tend to disregard the problem of cognitive authority. We do not customarily question major classifications or the cultural points of view represented in them because first and foremost we "know" they are "correct." We have been brainwashed by our own theoretical posture.

Of course we understand these classifications - the litany is constant: Dewey Decimal Classification, Library of Congress Classification, Universal Decimal Classification-to be derived from literary warrant. But we fail to analyze the authority by which that segment of literature is used as the major source of warrant for any 
particular KOS. Furthermore I wrote (Smiraglia 2016 forthcoming):

We also do not know what authority they bring to the table. We trust their editorial boards to behave according to societal norms. Many people believe that means that we trust them to behave objectively, but this is not correct. We expect, and therefore we receive, that the governance of classifications will remain faithful to the social epistemological role of disseminating the culture-no divergence from mainstream points of view, maintenance of mainstream thought, ongoing circulation of dominating ideas, and acquiescence if not obeisance of the receiving user community. Westerners, particularly North Americans, remain oblivious to these subtle influences from powerful KOSs.

KOSs, like $\mathrm{KO}$, are pervasive through time and culture, and serve not only to facilitate retrieval but also to constrain knowledge discovery. In fact, KOSs can be considered among information institutions, in which guise they ought then to be subject to cultural synergy. Specifically (Smiraglia 2014, 5):

It means simply that every information institution springs from within a culture, and therefore represents that culture from which it has sprung, but also can be found in alignment with other aligning cultures. [An information institution, such as a KOS] has, therefore, not a single cultural mission but many."

Every KOS is developed in response to the need of some identifiable domain, which is why there can be no true general or universal KOS. Context in $\mathrm{KO}$ is everything. The KO domain needs to begin to consider how the KOSs they support represent particular domain-centric representations of isolated unitary canonical literature, generally from western academic circles. These classifications then, have as a major goal the perpetuation and future dissemination of these particular, isolated, unitary and thus problematic canons of western academic culture.

\subsection{TWO QUESTIONS ABOUT KOS AS CULTURAL ARBITERS}

A first question for research is, how may we begin to interpret cultural pervasiveness of KOSs by considering their cultural roles and influences, ideological or otherwise? One answer is to take up explicit social epistemological analysis of KOSs. A nascent theory of engaged knowledge organization has emerged (Tennis 2013), in which KOSs are analyzed with regard to the unseen objective violence done to peoples and cultures that are misrepresented. One approach to the interpretation of harm has set a goal of recognizing "processes and implications" to raise "awareness" in the construction of KOSs in specific contexts and for specific constituencies (Adler and Tennis 2013, 270).

A second question is to what extent do KOSs, acting as stewards or vehicles of cultural dissemination, actually constrict the entry of new ideas? The antithetical question must also be posed-How is it that especially pervasive and long-lived KOSs close the gates to new, liberating, concepts? Collins (1998) suggested the presence of ongoing devices in knowledge domains by which competing points of view always are limited. If domains limit the breadth of viewpoints within them, then this limitation must accompany warranted KOSs arising from those domains as well. Of cultural dissemination, Smiraglia $(2014,50)$ wrote:

The culture must be permeated with its own seed, as it were, because the very survival of the culture demands it. The evolution and thriving of the culture depend on the dissemination of the information process. The gate swings both directions; gatekeepers open and close the gate, and consumers in a culture walk through the gate in both directions.

Pervasive KOSs always are involved in layers of interaction with many entities. For example, Elichirigoity and Malone (2005) demonstrate the interactive layers of North American economies in the North American Industry Classification System (NAICS). These include economic assumptions from neo-liberal economic theory that acted as gate-closers to new forms of production that emerged, such as open-source software, which they see as both a commercial product and a form of politics and freedom of expression. Fox (2015) traces the representations of sex and gender in the Dewey Decimal Classification to show that the blend of professional legal and medical discourse has been used to close the gate authoritatively on social and cultural understanding of existential reality.

Accustomed as the $\mathrm{KO}$ domain is to viewing any KOS as the ontological representation of sets of domain-centric concepts, the reality is that all conceptual entities in any domain are the products of multiple layers of discourse. Discourse is the realm of metaphorical conversations that inhabits the cultural foundations of any domain. Such conversations are rehearsed repeatedly in the canonical writings of a domain and of its precedent related domains that provide the cultural and social contexts of specific concepts. For example, sex can be perceived as the binary set \{male, female\} and gender can be perceived as the corresponding binary set \{masculine, feminine\}. Using Furner's (2007) terms, we might refer to these as natural 
kinds. But we might also take into consideration transsexuality or individuals who cross gender roles. Are these too natural kinds? Or are they nominal kinds? Or are they rendered as abnormality, over against the sets of natural kinds? Discourse makes these determinations and warrant ushers them into KOSs.

The products of discourse, then, are so absorbed by the users of a KOS that they become perceived as fact, convenience, or even obeisance. At any rate they might become perceived as natural kinds in the evolving discourse. Only on the rare occasions when shifts in domain theory cause revision of the discourse, which subsequently is expressed in new canonical texts, do the products of cultural or social change lead to change in the domain's KOSs.

More to the point of this essay is the fact that we have considered only one direction of discourse so far, although conversation should take place in two or more directions. As ideas and challenges arise from communities inhabiting domains they move through canonical discourse into a cultural canon and arrive in KOSs. Rarely, if ever, do shifting experiences of a community move through a KOS into the cultural discourse. It is here that the role being carved out by Fox, Furner, Elichirigoity and others, in which the KOS itself becomes a partner in the cultural discourse, can be seen as critical to the survival of $\mathrm{KO}$ as a science and a domain of application.

\subsection{CONCLUSION: A THIRD QUESTION ABOUT KOS AND ONTOGENY}

One last question is how to accompany the study of ontogeny (Tennis 2002; Salah et al. 2012) with analysis of societal effect over time. Temporality and atemporality meet in the canonical cultural discourses that govern KOSs. The conversation from source to KOS to user is always regarded as temporal or sequential. The study of subject ontogeny, generated by Tennis (2002) has so far sought to arrange the temporal sequences of evolving discourses enshrined in KOSs in sequence, with special care for the reverse order of changes to KOSs. In fact, the etymological implication of ontogenesis is the mapping of an entire lifetime.

Tennis' work shows the distorted path of eugenics among and along temporal social and cultural waves over more than a century as it moves from science to discredited social science to exile and back to a position of severe proscription as a concept with limited relevance in biology and genetics. Furner (2007) shows how concepts of race shift along the same temporal, social and cultural waves, as does Fox (2015) with sex and gender. Eugenics, race and sex and gender have been studied as elements of the discourse entrapped in the versions of the Dewey Decimal Classification. Salah et al. demonstrate the variability of a specific general bibliographic classification as the academic canon itself bulges and shifts over time. Their visualizations of change in the UDC create a virtual painting of the effect of cultural pervasiveness of classification.

But the atemporality of oppressive influence, which often is present implicitly or explicitly in canonical cultural discourse, so far has been overlooked. This brings us back to the concept in the opening of this paper: the pervasiveness of classification and its cultural affect need to be formally addressed in the knowledge organization domain.

\section{REFERENCES}

Adler, Melissa and Joseph T. Tennis. 2013. "Toward a Taxonomy of Harm in Knowledge Organization Systems." Knowledge Organization 40: 266-72.

Beghtol, Clare. 2004. "Naïve Classification Systems and the Global Information Society." In Knowledge Organization and the Global Information Society; Proceedings of the Eighth International ISKO Conference 13-16 July London UK, ed. Ia C. McIlwaine. Advances in Knowledge Organization v. 9. Würzburg: Ergon-Verlag, pp. 19-22.

Bowker, Geoffrey C. and Susan Leigh Star. 1999. Sorting Things Out: Classification and its Consequences. Cambridge, Mass.: MIT Press.

Collins, Randall. 1998. The Sociology of Philosophies: A Global Theory of Intellectual Change. Cambridge: Belknap.

Elichirigoity, Fernando and Cheryl Knott Malone. 2005. "Measuring the New Economy: Industrial Classification and Open Source Software Production." Knowledge Organization 32: 117-27.

Fox, Melodie J. 2015. Gender as an "Interplay of Rules:" Detecting Epistemic Interplay of Medican and Legal Discourse with Sex and Gender Classification in Four Editions of the Dewey Decimal Classification. Ph.D. Dissertation. University of Wisconsin, Milwaukee.

Furner, Jonathan. 2007. "Dewey De-Racialized: A Critical Race-Theoretic Perspective." Knowledge Organization 34: 144-68.

Jacob, Elin K. 2001. "The Everyday World of Work: Two Approaches to the Investigation of Classification in Context." Journal of Documentation 57: 76-99.

Lee, Hur-Li. 2016. Intellectual Activism in Knowledge Organization: A Hermeneutic Study of The Seven Epitomes. Taipei, Taiwan: National Taiwan University Press, forthcoming.

Mai, Jens Erik. 2011. "The Modernity of Classification." Journal of Documentation 67: 710-30.

Salah, Almilah Akdag, Cheng Gao, Kryzstof Suchecki, Andrea Scharnhorst and Richard P. Smiraglia. 2012. "The Evolution of Classification Systems: Ontogeny of the UDC." In Categories, contexts, and relations in 
knowledge organization: Proceedings of the Twelfth International ISKO Conference, 6-9 August 2012, Mysore, India, ed. A. Neelameghan and K.S. Raghavan. Advances in knowledge organization 13. Würzburg: Ergon Verlag, pp. 51-57.

Smiraglia, Richard P. 2014. Cultural Synergy and Information Institutions. New York: Springer.

Smiraglia, Richard P. 2016. "Foreword." In Intellectual Activism in Knowledge Organization: A Hermeneutic Study of The Seven Epitomes. Taipei, Taiwan: National Taiwan University Press, forthcoming.

Tennis, Joseph T. 2002. "Subject Ontogeny: Subject Access Through Time and the Dimensionality of
Classification." In Challenges in Knowledge Representation and Organization for the 21st Century: Integration of Knowledge across Boundaries: Proceedings of the Seventh International ISKO Conference. July 10-13, 2002, Granada, Spain, ed. Maria J. López-Huertas and Francisco J. MunozFérnandez. Advances in Knowledge Organization 8. Würzburg: Ergon Verlag, pp. 54-59.

Tennis, Joseph T. 2013. "Ethos and Ideology of Knowledge Organization: Toward Precepts for an Engaged Knowledge Organization." Knowledge Organization 40: 42-49. 